\section{Jet-like features near the nucleus of Chiron}

\author{
J. L. Ellot* ${ }^{*} \ddagger$, C. B. OlkIn*t, E. W. Dunham $\$$, \\ C. H. Ford $\|$, D. K. Gilmore, , D. Kurtz ${ }^{\#}$, \\ D. Lazzaro**, D. M. Rank $\rceil$, P. TemlT, \\ R. M. Bandyopadhyay*, J. Barroso**, A. Baruccl $\dagger$, \\ A. S. Bosh $\neq$, M. W. Bule + , S. J. Bus*, C. C. Dahn $+\neq$, \\ D. W. Foryta $\S$, W. B. Hubbard \|\| , D. F. Lopes**, \\ R. L. Marclalls \|\| $\mid$ T, S. W. McDonald*, R. L. Mlllisł, \\ H. Reltsema\#\#, D. G. Schleicher $\neq$, B. Slcardy $\dagger^{\dagger * * *}$, \\ R. P. S. Stone $+\dagger \dagger$ \&. L. H. Wasserman
}

CONSIDERED as a comet, the object 2060 Chiron is unusual in two respects: it exhibits outbursts at very large distances from the Sun $^{1-3}$, and its nucleus is much larger than that of any other known comet $^{4.5}$. It is, however, similar in size to the recently discovered Kuiper-belt objects ${ }^{6}$-a population of objects with orbits beyond Neptune, which are a possible source of short-period comets. This has led to the conjecture that Chiron is related to these objects, but its chaotic orbit has brought it much closer to the Sun ${ }^{7}$. Here we report observations of a recent stellar occultation by Chiron which permit the identification of several features associated with Chiron's coma. The observation of discrete, jet-like features provides evidence that the coma material originates from just a few, small active areas, rather than from uniform sublimation, and that the particles in at least one of these features have radii greater than $0.25 \mu \mathrm{m}$. The observations also suggest the presence of material in the plane of Chiron's orbit and are consistent with a gravitationally bound coma. Finally, the present data, and those from a previous occultation $^{8}$, constrain the radius of Chiron to lie between 83 and $156 \mathrm{~km}$.

The 9 March 1994 occultation of Ch08 (GSC248-01674) ${ }^{9}$ by Chiron was successfully observed with two telescopes: the $0.9-\mathrm{m}$ telescope aboard NASA's Kuiper Airborne Observatory (KAO) flying near Recife, Brazil and the $0.5-\mathrm{m}$ telescope at the South African Astronomical Observatory (SAAO) at Sutherland. We attempted observations with five other telescopes in Brazil. But their positions turned out to be far from the final predicted shadow path of Chiron, and all were clouded out. Instrumental parameters for the observations are specified in Table 1. Compared with its photometric history ${ }^{2}$, Chiron was relatively faint at the time of our observations, with a standard magnitude ${ }^{10}$ $H_{\mathrm{v}}=6.6 \pm 0 . \mathrm{I}$.

We generated a light curve from the KAO optical data using the technique of point-spread-function (PSF) model fitting. The infrared light curve was produced by doing aperture photometry with a synthetic aperture three pixels in radius (image radii ranged between 1.0 and 2.0 pixels). The SAAO light curve was obtained directly from the recorded photomultiplier-tube integrations. Sections of the light curves that bracket Chiron's closest approach to the star are shown in Fig. I. A sharp drop and recovery occurs in all light curves, which we shall denote as feature 1 (F1). This appears similar to a feature in the Palomar light curve for the earlier Chiron occultation-one

\footnotetext{
- Department of Earth, Atmospheric and Planetary Sciences, Massachusetts Institute of Technology, Cambridge, Massachusetts 02139-4307, USA † Department of Physics, Massachusetts Institute of Technology Cambridge Massachusetts $02139-4307$ USA t Lowell Observator institu

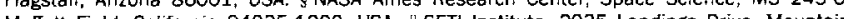
Mofett Feld, Calforia $94035-1000$, USA. I SET lnstute, 2035 Landings Drive, Mountain View, California 94043, USA. University of California, Lick Observatory, Santa Cruz, California 95064, USA. \# University of Cape Town, Private Bag, Rondebosch 7700, Cape Town, South Africa. ${ }^{* *} \mathrm{CNPq} /$ Observatório Nacional, DAF, 20921 Rio de Janeiro, Brazil. ++ Obsenvatoire de Paris, 92195 Meudon Cédex Principal, France. ++ US Naval Observatory, PO Box 1149, Flagstaff, Arizona 86002 1149, USA. W UFPR/Dep. Fisica, Centro Politecnico, 80000 Curitiba, Brazil. III Lunar and Planetary Laboratory, University of Arizona, Tucson, Arizona 85721, USA. $\because$ Pima Community College, 2202 West Anklam Road, Tucson, Arizona 85709, USA \#\# Ball Aerospace. PO Box 1062 , Bouler, Colo Wo 75252 Paris Cedex 05, France. ${ }^{++}+$Lick Observatory, Mount Hamilton, California 95140, USA.
}

interpretation of which is a dust jet ${ }^{8}$. In addition, broader and shallower occultation features are particularly evident in the KAO optical light curve. To show the occultation features more clearly, shorter sections of the light curves are shown in Fig. I, right panel.

In addition to $\mathrm{Fl}$ in the $\mathrm{KAO}$ optical light curve, we also see a broader feature (F2), that has a full-width at half-maximum (FWHM) of $\sim 74 \mathrm{~km}$, but less optical depth. Due to lower signalto-noise ratio, F2 is not detectable in the infrared or SAAO light curves (although there is a hint of it in the former). Also, we note in the KAO optical light curve a low-level attenuation of the stellar signal that begins at $\sim 517 \mathrm{~s}$ after the reference time and a similar attenuation between FI and F2. Noting that F2 has a steeper slope on its leading edge than its trailing edge, one could interpret $\mathrm{F} 2$ as a symmetrical feature embedded in a lowlevel attenuation that ends at the trailing edge of F2. Without knowing how to apportion the optical depth into real, physical features, we have taken the liberty of labelling $F 3$ as a feature that begins with the first low-level attenuation and ends at the trailing edge of F2. Finally, a feature labelled F4 is present in the KAO optical light curve. Its significance is $3 \sigma$ (well below the detection limits of Earth-based imaging), and it occurs when the star aligns with Chiron's orbit plane, where material may be expected. Table 2 gives information about the optical depths and widths of the features.

To establish the geometrical relationship of these features in the light curves to the nucleus of Chiron, in Fig. 2 we have plotted a Chiron-centred sky frame "that shows the orbit plane, the track of the star from both stations, and the direction to the Sun. Because Chiron was only $15^{\circ}$ from opposition, the largest component of the vector to the Sun is actually perpendicular to the plane of Fig. 2. The relative position of the star and Chiron's centre of light (assumed to be the centre of the nucleus) was determined from 40 astrometric frames taken with the 61-inch astrometric reflector at the US Naval Observatory, which yielded formal errors $(1 \sigma)$ of $\pm 16 \mathrm{~km}$ in right ascension and declination (the $f, g$ coordinate systems in Fig. 2). The broad, low-opticaldepth feature (F3) is nearly symmetrical around the closest approach time of the event, and F4 occurs near the orbit-plane crossing for the KAO (at 575 $\pm 5 \mathrm{~s}$ after 23:20:01 UT- the uncertainty is larger than the uncertainty in the closest approach owing to the oblique intersection of the apparent star path with the orbit plane).

We can argue against the possibility that $F 1$ is the signature of a small, sharp-edged body or that of a grazing occultation by the nucleus. Although a small body (or the nucleus) could produce a partial occultation, its characteristic dimensions would be of the order of the Fresnel diffraction scale $\sqrt{\lambda D}$, where $\lambda$ is the wavelength of observation and $D$ is the body observer distance. These values are 0.9 and $1.6 \mathrm{~km}$ for the optical and infrared data respectively (the projected angular diameter of the star at Chiron's distance would be even less, only $0.1 \mathrm{~km}$ ). The wings of $F 1$ in the KAO optical light curve are much broader than this, ruling out a solid body or nuclear occultation (unless either of these were surrounded by a dust cloud that produced the wings).

Accepting that $F 1$ is not a partial nuclear occultation, we can place a $3 \sigma$ upper limit on Chiron's radius of $156 \mathrm{~km}$ from our closest approach distance of $108 \pm 16 \mathrm{~km}$. Compare this with the $83 \mathrm{~km}$ lower limit on Chiron's radius derived from the previous stellar occultation ${ }^{8}$ and radii ranging between $74 \pm 11 \mathrm{~km}$ and $104 \pm 10 \mathrm{~km}$ from thermal infrared observations ${ }^{5}$.

Other possibilities for F1 would be a suborbital arch, dust jet. or shell-the last two of which are seen in the comae of comets ${ }^{i 2}$ and emanating from the nucleus of comet Halley ${ }^{13,14}$. This interpretation would be consistent with the smooth appearance of the coma from ground-based imaging ${ }^{15}{ }^{17}$, as all features we see would not be resolved in those data. The interpretation of F1 as caused by the outflow from a conical jet requires a spreading angle of $\sim 12^{\circ}$ for our nominal geometrical solution and a nuclear 
FIG. 1 Chiron occultation light curves. Portions of the stellar flux (normalized to 1.0 for the unocculted star) from Ch08 have been plotted versus UT for an interval that includes Chiron's closest approach to Ch08 (shown by the dotted line labelled "CA"). The lefthand panel is at full resolution. The right-hand panel includes only data near closest approach; to enhance the signal-to-noise ratio, data outside the sharp drop (feature 1, F1) have been averaged to $2.0 \mathrm{~s}$. The segment of the light curves comprising $F 1$ have been left at full resolution for the KAO data, whereas the SAAO data have been
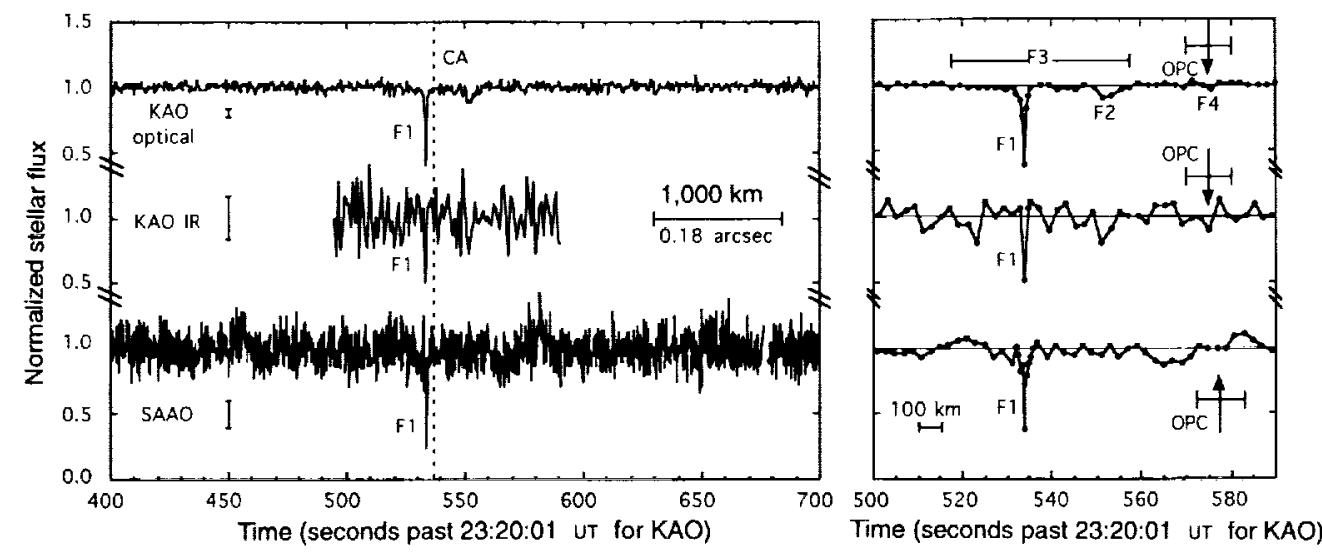
averaged to $0.4 \mathrm{~s}$ (two integration

times and two deadtimes). In the left-hand panel the error bars are $\pm 1 \sigma$, and the $1,000 \mathrm{~km}$ spatial scale at Chiron (for the $\mathrm{KAO}$ shadow velocity of $18.32 \mathrm{~km} \mathrm{~s}^{1}$ ) is approximately the resolution of the Hubble Space Telescope. The orbit-plane crossing (OPC) time is indicated in the right-hand panel. A sharp drop and recovery (F1) is evident in all three light curves. As the paths of the KAO and SAAO were separated in Chiron's shadow plane by only $1.4 \mathrm{~km}$ at closest approach $(107.8 \mathrm{~km}$ for the KAO and $106.4 \mathrm{~km}$ for $\mathrm{SAAO}$ ), we have assumed that $\mathrm{F} 1$ was

caused by the same material for both stations. We therefore corrected for the unknown timing offset in the SAAO data by retarding the time axis of the light curve by $309.9 \mathrm{~s}$ to align the sharp feature with that of the KAO. Near $680 \mathrm{~s}$, there is a gap in the SAAO light curve, where a noise spike was removed. These spikes reached a flux level $100 \%$ above the ChO8 level and were removed before calculating the unocculted level for ChO8. See text for description of the features.

radius of $83 \mathrm{~km}$ (ref. 8), if we assume that the jet originates on the limb in the sky plane and from a small active area on the surface - consistent with the $1-2 \mathrm{~km}$ radii for active areas that would have been required to produce the visible coma at earlier times $^{3,16}{ }^{18}$. Larger and smaller spreading angles are possible for other assumptions.

The ratio of extinction at infrared to that at optical wavelengths for $F 1$ is formally $1.31 \pm 0.65$ (over equal integration intervals of $1.0 \mathrm{~s}$ ). For the particle-size distributions found for comet Halley ( $n^{\alpha} \mathrm{d} n$, with $\alpha$ between -4 for particles with radii of $\sim 100 \mu \mathrm{m}$ and -1.54 for those with radii $\sim 0.1 \mu \mathrm{m})^{19}$, this ratio would be much less than one. Hence the extinction in Fl can not be caused by this type of particle-size distribution, but must be dominated by large particles. To obtain some quantitative estimate of the lower limit on the particle size, we use Mie theory for spherical particles ${ }^{20}$, and find that for various compositions from silicate to tholin to graphite these data are consistent with a lower limit of $0.25 \mu \mathrm{m}$ (ref. 21 ) on the radius of particles. So most of the extinction we measure must be contributed by particles larger than this, but somewhat different limits would apply for non-spherical particles, with indices of refraction different from our assumption ${ }^{20}$. This conclusion for $F 1$ shows a particle population differing from that measured ${ }^{22}$ by the particle detectors on the Halley spacecraft, which found a significant population of particles with radii smaller than $0.25 \mu \mathrm{m}$. The material probed here, however, is from a different object, much further from the Sun, and sampled much closer to the nucleus. An upper limit to the radii of particles that can be lifted from the nucleus by escaping gas has been estimated ${ }^{17}$ at $100 \mu \mathrm{m}$ for $\mathrm{CO}$ and $10 \mu \mathrm{m}$ for $\mathrm{CO}_{2}$

\begin{tabular}{|c|c|c|c|c|c|c|c|}
\hline $\begin{array}{l}\text { Telescope } \\
\text { (diameter) }\end{array}$ & Detector & Filter & $\begin{array}{c}\text { Recording } \\
\text { interval (UT) }\end{array}$ & $\begin{array}{c}\text { Number of } \\
\text { integrations }\end{array}$ & $\begin{array}{l}\text { Integration } \\
\text { time }(\mathrm{s})\end{array}$ & $\begin{array}{c}\mathrm{S} / \mathrm{N} \\
\text { (for } 1 \mathrm{~s} \text { ) }\end{array}$ & $\begin{array}{c}\mathrm{S} / \mathrm{N} \\
\text { (for } 10 \mathrm{~km} \text { ) }\end{array}$ \\
\hline $\mathrm{KAO}(0.9 \mathrm{~m})$ & $\mathrm{CCD}$ & None* & $\begin{array}{l}23: 20: 01- \\
23: 36: 40.5\end{array}$ & 2,000 & 0.5 & 52 & 38 \\
\hline $\mathrm{KAO}(0.9 \mathrm{~m})$ & HgCdTe array & $k$ & $\begin{array}{l}23: 20: 01- \\
23: 36: 40.5\end{array}$ & 1,000 & 1.0 & 5.8 & 4.3 \\
\hline SAAO $(0.5 \mathrm{~m})$ & PMT & v & $\begin{array}{l}22: 58: 53- \\
23: 42: 40 t\end{array}$ & 13,266 & $0.1 \dagger$ & 20 & 15 \\
\hline
\end{tabular}

The occulted star is the brighter of two stars that have the combined photometric measurements: $V=11.95 \mathrm{mag},(B-V)=0.92,(V-R)=0.42$, $(V-I)=0.86,(V-K)=1.9$. The fainter companion star lies at a distance of 3.27 arcsec from the brighter one, at a position angle of $19.6^{\circ}$, and it has magnitude differences with ChO8 of 2.15 in the $K$ band and 3.83 in a broadband red filter $(6,600-8,300 A)$, used by the US Naval Observatory. The KAO optical data were recorded with a high-speed CCD (charge-coupled device) photometer ${ }^{24}$, using two $60 \times 60$ pixel subframes $(0.98$ arcsec per pixel): one subframe contained the merged images of Chiron, Ch08 and the faint companion star; the other contained a field star of brightness comparable to Ch08 and 4.38 arcmin away. A continuous series of 2,000 subframe pairs were recorded, and the timing of the series was related to UT with a GPS clock. A beamsplitter enabled simultaneous infrared observations to be carried out with a NICMOS HgCdTe array $(128 \times 128$ pixels, $\sim 1.4$ arcsec per pixel) that was read out once per second, synchronously with the optical data recording. The SAAO data were recorded with a photomultiplier tube (PMT) at an integration time of $0.1 \mathrm{~s}$, through an aperture 30 arcsec in diameter. Reference to the standard star HR4064 showed that the night was photometric at SAAO. The deadtime between integrations was usually $0.1 \mathrm{~s}$, but, sometimes it was $0.0 \mathrm{~s}$, which made it impossible to accurately relate the recording times of these data to ur. The signal-to-noise ratio $\mathrm{S} / \mathrm{N}$ in the next to last column is defined as the ratio of the unocculted flux of the star to the r.m.s. noise, each integrated for one second. The last column contains the analogous ratio for a $10 \mathrm{~km}$ interval in the sky-plane.

* The unfiltered CCD response and stellar spectrum combine for a mean wavelength of $\sim 0.68 \mu \mathrm{m}$.

t The end time of the SAAO data is not precisely known. The value here was calculated for a deadtime of $0.1 \mathrm{~s}$ between integrations for $98 \%$ of the data, and no deadtime between the remaining $2 \%$ of the integrations (as the deadtime between successive integrations was $0.1 \mathrm{~s}$ about $98 \%$ of the time and $0.0 \mathrm{~s}$ for the remaining $2 \%$ ). 
FIG. 2 The Chiron-centred sky-plane. Chiron's shadow at Earth is indicated in the $(f, g)$ coordinate system ${ }^{11}$, in which $f$ is parallel to right ascension and $g$ is parallel to declination. The orbit plane of Chiron has been plotted for reference. Along the paths probed by Ch08 as seen from the KAO and SAAO, relevant times for light-curve features are indicated, and time increases to the southeast. Note that the occultation probed the orbit plane ahead of Chiron. Along the apparent path of the star, we have indicated the positions of the features derived from the KAO optical light curve. Coincidentally both stations probed nearly the same region near Chiron. The uncertainty in the closest approach time is the geometrical error divided by the shadow velocity $(\sim 0.9 \mathrm{~s})$. Chiron was $15^{n}$ from opposition at the occultation time, so the vector to the Sun is mostly out of the plane of the figure. A Chiron radius of $83 \mathrm{~km}$ has been assumed ${ }^{8}$ (indicated by the shaded circle). For the KAO, the occultation shadow probes the orbit plane at $575 \mathrm{~s}$ after 23:20:01 UT, and there is a $3 \sigma$ occultation feature evident in the light curve at this time (F4). If $F 1$ and $F 2$ are caused by jets originating from the limb of the nucleus nearest to where these features were detected, then extending lines from F1 and F2 to Chiron's centre, we see that F2 would be on the side of the nucleus facing the Sun (consistent with the jet

If one interprets $F 1$ as a jet, it is natural to interpret $F 2$ as a jet also. Being probed further from the nucleus than FI, it has spread into a broader morphology. In fact, it is possible that FI and $F 2$ are caused by a single structure - shaped as a spiral or suborbital arch - that has been probed twice. In order to test this hypothesis, one would need to know the rotation of the nucleus. Although the period has been measured quite accurately ${ }^{2.3 .17}$, the direction of the rotation pole ${ }^{23}$, estimated from the imaged coma morphology, disagrees somewhat with constraints from photometry ${ }^{2}$. Because we are seeing these features projected onto a plane, however, their distances from the nucleus in this plane are only lower limits on their true distances. Furthermore, the vector to the Sun points mostly out of the sky plane, so that either F1 or F2 could be emanating from a sunlit or dark side of the nucleus. Despite these uncertainties, we presently feel that $F 1$ and $F 2$ are probably caused by two distinct dust jets from the nucleus.
Although F3 is much broader, it could possibly be a jet, shell, or other shape ${ }^{12}$ - seen much further from the nucleus than Fl or F2, but appearing nearly symmetrical with respect to the nucleus owing to projection effects. Another, and perhaps more plausible, explanation for F3 is that it is a symmetrical cloud of dust around the nucleus. If this material is flowing out uniformly at the speed of sound for $\mathrm{CO}\left(160 \mathrm{~m} \mathrm{~s}^{-1}\right)^{17}$, then the material rate of loss (for particles $10 \mu \mathrm{m}$ in radius and density $1 \mathrm{~g} \mathrm{~cm}^{-3}$ ) would be several orders of magnitude larger than that needed to account for the extended coma (in 1990, when Chiron was at least 0.5 mag brighter in $\left.H_{\mathrm{V}}\right)^{16,17,23}$. Therefore we believe that the material causing F3 is not flowing outwards from the nucleus at the speed of sound, but is possibly gravitationally bound to the nuclcus-a bound coma ${ }^{16,17}$. Such a feature would be unique to Chiron because it is the only known comet large enough for gravity to hold dust in bound orbits for periods ranging from tens of days ${ }^{18}$ to a year ${ }^{16}$. If $F 3$ is a manifestation of a bound

\begin{tabular}{|c|c|c|c|c|c|c|c|c|}
\hline Feature & Light curve & $\begin{array}{c}\text { Midtime } \\
\text { (s after } \\
23: 20: 01 \text { UT) }\end{array}$ & $\begin{array}{c}\text { Distance } \\
\text { from Chiron's } \\
\text { centre }(\mathrm{km})^{*}\end{array}$ & $\begin{array}{l}\text { Averaging } \\
\text { interval } \\
(\mathrm{km})\end{array}$ & $\begin{array}{l}\text { Maximum } \\
\text { optical depth } \\
\text { measured }\end{array}$ & $\begin{array}{l}\text { FWHM } \\
(\mathrm{km})\end{array}$ & $\begin{array}{c}\text { Integrated } \\
\text { optical depth } \\
(\mathrm{km})^{\dagger}\end{array}$ & Morphology \\
\hline 1 & $\begin{array}{l}\text { KAO, optical } \\
\text { KAO, infrared } \\
\text { SAAO }\end{array}$ & 533.7 & $126 \pm 16$ & $\begin{array}{r}9.2 \\
18.3 \\
7.3\end{array}$ & $\begin{array}{l}0.92 \pm 0.07 . \ddagger \\
0.68 \pm 0.34 \\
0.98 \pm 0.46\end{array}$ & $\begin{array}{l}5.5-9.2 \\
9.0-18.3 \\
4.5-7.3\end{array}$ & $\begin{array}{r}13.2 \pm 1.3 \\
12.4 \pm 6.2 \\
7.2 \pm 3.4\end{array}$ & $\begin{array}{l}\text { Narrow, deep, } \\
\text { symmetrical }\end{array}$ \\
\hline 2 & KAO, optical & 552.4 & $295 \pm 16$ & Model fit & $0.11 \pm 0.02$ & $74 \pm 15$ & $4.1 \pm 1.3$ & Asymmetric? \\
\hline 3 & KAO, optical & 537.4 & $108 \pm 16$ & Model fit & $0.027 \pm 0.006$ & $513 \pm 169$ & $21.6 \pm 5.0$ & Broad, flat \\
\hline 4 & KAO, optical & 575.7 & $712 \pm 16$ & Model fit & $0.07 \pm 0.04$ & $12 \pm 10$ & $1.6 \pm 0.5$ & $3 \sigma$ \\
\hline
\end{tabular}

The averaging interval is equal to the product of the apparent shadow velocity from the site and the integration interval for the data, and the maximum measured optical depth is derived from the integration having the lowest signal level. The differences between the integrated optical depth in the SAAO and KAO optical light curves can be attributed to one or more of the following: (1) the core of feature (F1) is not resolved in the KAO optical light curve, because the integration time is longer than for SAAO, (2) the duty cycle of the SAAO data recording is $50 \%$, whereas that for the KAO data is $100 \%$, or (3) F1 changed during the $310 \mathrm{~s}$ that elapsed between the recording of $\mathrm{F} 1$ at the two stations. The maximum optical depths and widths of F2, F3 and F4 were determined from fitting a lorentzian model.

* Distance of feature from Chiron's centre probed at midtime (column 3 ).

t The integrated optical depths depend on how one chooses to separate the features. For features 1 and 2 (F1 and F2) of the KAO optical light curve, the integrated optical depth was determined by taking the logarithm of the summed normalized stellar flux for all points in the feature (after correcting for the optical depth per point from $\mathrm{F} 3$ of 0.027). This optical depth was then converted to kilometres by multiplying by the velocity and integration time. For F3 the integrated optical depth was determined by model fitting (to include the optical depth contribution when this feature is embedded in the other features). The integrated optical depth for F4 was also determined by model fitting. Finally, the integrated optical depths for the KAO infrared and the SAAO data were calculated by multiplying the maximum optical depth by the averaging interval, with the SAAO data averaged over two points (for a time interval of $0.4 \mathrm{~s}$, including the deadtime).

$\ddagger$ Optical depth averaged over $18.3 \mathrm{~km}$ of projected width for feature 1 is $0.52 \pm 0.03$. This average should be used when comparing with the average optical depth in the infrared. The extinction ratio (infrared to optical) is $1.31 \pm 0.65$. 
coma, it may be only a small part, with the remainder having too low an optical depth to be detectable in these data.

Received 15 August; accepted 24 November 1994.

1. Tholen, D. J., Hartmann, W. K. \& Cruikshank, D. P. IAU Circ. No. 4554 (1988)

2. Marcialis, R. L. \& Buratti, B. J. icarus 104, 234-243 (1993).

3. Bus, S. J., Bowell, E., Harris, A. W. \& Hewitt, A. V. Icarus 77, 223-238 (1989)

4. Sykes, M. V. \& Walker, R. G. Science 251, 777-780 (1991).

5. Campins, H. et al. Astr. J. 108, 2318-2322 (1994).

6. Jewitt. D. \& Luu, J. Nature 382, $730-732$ (1993)

7. Scholl, H. lcarus 40, 345-349 (1979).

B. Buie, M. W. IAU Circ. No. 5898 (1993).

9. Bus, S. J., Wasserman, L. H. \& Elliot, J. L. Astr. J. 107, 1814-1824 (1994).

10. Bowell, E. et al. in Asteroids II (eds Binzel, R. P., Gehrels, T. \& Matthews, M. S.) (Univ. Arizona Press, Tucson, 1989)

11. Elliot, J. L. et at. Astr. J. 106, 2544-2572 (1993).

12. Sekanina. Z. in Comets in the Post.Halley Era (eds Newburn, R. L., Neugebauer. M. \& Rahe, J) 769-823 (Kluwer, Dordrecht, 1991).

13. Sagdeev, R. Z. et al. Nature 321, 262-266 (1986).

14. Sekanina, Z. \& Larson, S. M. Nature 321, 357-361 (1986)

15. West, R. M. Astr. Astrophys. 241, 635-645 (1991)

16. Meech, K. J. \& Belton, M. J. S. Astr. J. 100, 1323-1338 (1990)

17. Luu, J. X. \& Jewitt, D. C. Astr. J. 100, 913-932 (1990)

18. Stern, S. A., Jackson, A. A. \& Boice, D. C. Astr. J. 107, 765-771 (1994).

19. Lamy, P. L., Grūn, E. \& Perrin, J. M. Astr. Astrophys. 187, 767-773 (1987)

20. van de Mulst, H. C. Light Scattering by Small Particles (Dover, New York, 1981).

21. Olkin C B et at in Proc. Airborne Astronomy Symp on the Galactic Ecosystem: From Gas to Stars to Dust (eds Haas. M. R.. Davidson, J. A. \& Erickson, E. F.) (Astr. Soc. Pacif., San Francisco, 1994).

22. McDonnell, J. A. M., Lamy, P. L. \& Pankiewicz, G. S. in Comets in the Post-Halley Era (eds

Newburn, R. L., Neugebauer, M. \& Rahe, J.) 1043-1073 (Kluwer, Dordrecht, 1991)

23. Fulle, M. Astr. Astrophys. 282, 980-988 (1994).

24. Buie, M. W. et al. Bull. Am. Astro. Soc. 25, 1115 (1993).

ACKNOWLEDGEMENTS. We thank the entire KAO crew for their outstanding work, especially mission director $\mathrm{J}$. McClenahan and navigator $\mathrm{R}$. Morrison; we also thank the members of the Brazilian and US governments who worked out agreements that allowed KAO to use Brazilian Brazilian and US governments who worked out agreements that allowed KAO to use Brazilian air space and airport accommodation on short notice. The NICMOSII FPA used in these observaby NASA 